\title{
AMAMANTAMIENTO RESTRINGIDO Y SUPLEMENTACIÓN SOBRE LOS PERFILES METABÓLICOS EN VACAS DEL SISTEMA DOBLE PROPÓSITO
}

\section{RESTRICTED SUCKLING AND SUPPLEMENTATION ON THE METABOLIC PROFILES IN COWS OF THE DOUBLE PURPOSE SYSTEM}

\author{
Roger Salgado O*, Lino Torregroza S, Jaime Álvarez P, Nicolás Martínez H, Clara Rúgeles P, \\ Ó scar Vergara G, Libardo Maza A, G uillermo Martínez F.
}

Universidad de Córdoba, Facultad de Medicina Veterinaria y Zootecnia, Departamento de Ciencias Pecuarias. A.A. 354 Montería, Colombia. *Correspondencia: rsalgado@ sinu.unicordoba.edu.co

\author{
Recibido: Marzo 28 de 2006; Aceptado: 0 ctubre 26 de 2006
}

\section{RESU MEN}

O bjetivo. Evaluar el efecto de la suplementación con semilla de algodón y el sistema de amamantamiento sobre los perfiles metabólicos desde el parto hasta la semana 14 posparto. Materiales y métodos. Se conformaron 4 tratamientos: T1 con 4 vacas suplementadas con 2 kilos de semilla de algodón y amamantamiento experimental; T2 con 3 vacas suplementadas con 2 kilos de semilla de algodón y amamantamiento tradicional; T3 con 3 vacas sin suplemento y amamantamiento experimental; T4 con 3 vacas sin suplemento y amamantamiento tradicional. Se utilizó un diseño al azar con arreglo factorial $2 \times 2$ y para el análisis de resultados se empleó análisis de varianza. Resultados. En el posparto, las vacas suplementadas presentaron mayores niveles $(p<0.05)$ de proteína y de colesterol en sangre en la quincena 7 ; igualmente mostraron mayores niveles de triglicéridos y HDL en las quincenas 2 y 4 respectivamente que las vacas no suplementadas. Hubo efecto del amamantamiento sobre la proteína en las quincenas 3, 6 y 7 $(p<0.05)$; se encontraron mayo res concentraciones en los animales con amamantamiento tradicional versus amamantamiento restringido (91.2 y 63.8; 94.2 y 71.1 y 95.8 y $71 \mathrm{~g} / \mathrm{l}$, respectivamente). Hubo correlación $(p<0.05)$ de los tratamientos sobre los triglicéridos durante las quincenas 2,6 y 7. No hubo efecto $(p>0.05)$ de los tratamientos sobre la reproducción posparto. Conclusión. La suplementación con semilla de algodón no tuvo efecto sobre los perfiles metabólicos durante el preparto. En el postparto la suplementación y el amamantamiento tuvieron efecto en algunos metabolitos, principalmente triglicéridos y proteínas.

Palabras clave: Amamantamiento restringuido, metabolismo, semillas de algodón, bovinos, doble propósito.

\section{ABSTRACT}

0 bjective. To evaluate the effect of supplementation with whole cotton seed and the suckling system on the metabolic profiles from calving until the week 14 postpartum. Materials and methods. Four 
treatments were conformed: T1 with 4 cows supplemented with $2 \mathrm{~kg}$ of whole cotton seed and experimental suckling; T2 with 3 cows supplemented with $2 \mathrm{~kg}$ of whole cotton seed and traditional suckling; T3 with 3 cows without supplement and experimental suckling; T4 with 3 cows without supplement and traditional suckling. A complete random design with a $2 \times 2$ factorial arrangement was used and for the result analysis an AN 0 VA was used. Results. In the postpartum, the supplemented cows presented highest levels of protein $(p<0.05)$ and cholesterol in blood in the fifteenth period 7 ; equally highest levels of triglycerides and HDL showed in the fifteenth periods 2 and 4 respectively that the cows non supplemented. There was suckling effect on the protein in the fifteenth periods 3 , 6 and $7(p<0.05)$; There were bigger in the animals with traditional suckling versus restricted suckling (91.2 vs $63.8 ; 94.2$ vs 71.1 and 95.8 vs $71 \mathrm{~g} / \mathrm{l}$, respectively). There was correlation $(p<0.05)$ of treatments on triglycerides during the fifteenth periods 2, 6 and 7 . There was not effect $(p>0.05)$ of the treatments on the postpartum reproduction. Conclusion. During prepartum supplementation with whole cotton seed did not have effect on the metabolic profiles. In the postpartum the supplementation and suckling had effect in some metabolites, mainly triglycerides and proteins.

Key words: Restricted suckling, metabolism, cotton seeds, bovine, double purpose.

\section{INTRODUCCIÓN}

El período de transición de la vaca se refiere al tiempo aproximado de 3 semanas antes y 3 semanas después del parto y es la fase más crítica del ciclo de la lactancia (1). Este período es caracterizado por una disminución en la ingesta de alimento (2), aumento en la movilización de tejidos grasos y elevación de ácidos grasos no esterificados en sangre $(3,4)$. También, se refiere a la movilización proteica y cambios hormonales relacionados con el parto y lactancia (5). Lo anterior puede desarrollar desórdenes metabólicos y una menor eficiencia reproductiva y productiva postparto (6).

G rummer et al. (1), reportaron que el efecto de la suplementación proteica durante el preparto y postparto temprano no ha sido adecuadamente investigado. Sin embargo, en la literatura se encuentran resultados contradictorios. Putnam et al. (7) y Van Saun et al. (8) encontraron un mejoramiento tanto en el estado metabólico como en la condición corporal de la vaca, mientras que otros como Grieenfield et al. (3) y Doepel et al. (9) reportan que aumentando el nivel de proteína en ganado de leche durante el período de transición no se mejora el desempeño reproductivo en la subsecuente lactación. Con relación al amamantamiento, también se han presentado resultados contradictorios, algunos autores confirman el efecto del amamantamiento sobre la pérdida de peso y condición corporal (10 - 12) mientras que otros no han encontrado ese efecto (13 - 19).
El objetivo de este estudio fue el de determinar el efecto de la suplementación proteica y el tipo de amamantamiento sobre los perfiles metabólicos en vacas F1 Holstein x Brahman.

\section{MATERIALES Y MÉTODOS}

Sitio de estudio, geoclima, grupos de bovinos $y$ tratamientos. El estudio fue realizado en una finca localizada en la vereda Canta Rana, municipio de San Carlos (Córdoba), ubicada a $8^{\circ} 45^{\prime}$ latitud norte y $75^{\circ}, 22^{\prime}$ longitud oeste, altura de $15 \mathrm{msnm}$, precipitación media anual de $1100 \mathrm{~mm}$, temperatura media anual de

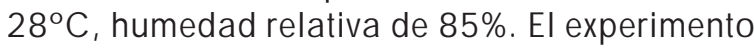
se llevó a cabo durante el perío do comprendido entre el mes de septiembre de 2004 hasta el mes de abril de 2005 .

Se utilizaron 13 vacas de primer parto, $\mathrm{F} 1 \mathrm{HxC}$, con una condición corporal al parto de 4 evaluada en un escala de 1 a 5 . Después del parto, las vacas y sus respectivos terneros fueron distribuidos en cuatro grupos de tratamiento según un arreglo factorial $2 \times 2$ (alimentación $x$ amamantamiento) a saber:
$\mathrm{T} 1$ = Vacas suplementadas $\mathrm{y}$ amamantamiento experimental
$\mathrm{T} 2=$ Vacas suplementadas $\mathrm{y}$ amamantamiento tradicional
$\mathrm{T3}=$ Vacas no suplementadas y amamantamiento experimental
$\mathrm{T4}=$ Vacas no suplementadas $\mathrm{y}$ amamantamiento tradicional 
El amamantamiento experimental consistió en amamantar las crías dos veces al día, durante 30 minutos, entre las 6 a 8 y a las 13 a 14 horas. Una vez terminado el amamantamiento, los terneros fueron separados de las madres y llevados a potreros. En el amamantamiento tradicional, los terneros, después del ordeño, pastoreaban con sus madres hasta las 13 a 14 horas, para luego ser separados y llevados a potreros diferentes. El suplemento consistió en el suministro de 2 kilogramos de semilla de algodón por animal durante el período experimental.

Los grupos experimentales fueron manejados bajo un sistema rotacional de praderas con un período de descanso de 24 días, un período de permanencia de un día, con disponibilidad de agua y sal mineralizada a voluntad. Durante la época seca, todos los animales en estudio recibieron ensilaje de pasto angleton (Dichanthium aristatum) a razón de 10 Kilos/ animal/día además del pastoreo.

Toma de muestras y análisis. De cada animal después del parto, en la mañana se obtuvo semanalmente hasta la semana 14 postparto,
$10 \mathrm{ml}$ de sangre sin anticoagulante por venopunción coccígea antes de ser ofrecido el suplemento. Las muestras fueron llevadas al laboratorio de reproducción de la Facultad de Medicina Veterinaria y Zootecnia de la Universidad de Córdoba, donde fueron centrifugadas a $3000 \mathrm{rpm} \times 10$ minutos para separar el suero. Posteriormente se conservaron a $-20 \cong 0$ hasta su análisis.

Química sanguínea. Del total de muestras obtenidas se determinó la concentración de glucosa, colesterol, colesterol HDL, colesterol $\mathrm{LDL}$, triacilgliceroles, urea, nitrógeno ureico en sangre (BUN) y proteína total en sangre. En la tabla 1 se presentan los métodos analíticos que se emplearon, así como el sistema de unidades internacionales (SIU) para cada una de las variables analizadas.

Análisis estadístico. Se utilizó un diseño al azar, con un arreglo factorial $2 \times 2$, con dos sistemas de alimentación y dos sistemas de amamantamiento, con 3 repeticiones por grupo experimental. Los datos fueron analizados por el procedimiento GML de SAS (Institute Inc., Cary, NC).

Tabla 1. Métodos analíticos empleados para la determinación de perfiles metabólicos.

\begin{tabular}{lcl}
\hline Variable & Unidades (SIU)* & \multicolumn{1}{c}{ Método } \\
\hline Glucosa & $\mathrm{mmol} / \mathrm{l}$ & GOD-PAP \\
Colesterol & $\mathrm{mmol} / \mathrm{l}$ & CHOD-PAP \\
Colesterol-HDL & $\mathrm{mmol} / \mathrm{l}$ & Fosfotungstato magnesio-CHOD-PAP \\
Colesterol LDL & $\mathrm{mmol} / \mathrm{l}$ & Ecuación de Friedewald \\
Triacilgliceroles & $\mathrm{mmol} / \mathrm{l}$ & Peroxidasa-oxidasa de G licerol Fosfato \\
Urea & $\mathrm{mg} / \mathrm{dl}$ & Ureasa \\
Bun & $\mathrm{mg} / \mathrm{dl}$ & Ureasa \\
Proteínas totales & $\mathrm{g} / \mathrm{l}$ & Biuret \\
\hline
\end{tabular}

*SIU: Sistema Internacional de Unidades

\section{RESULTADOS Y DISCUSIÓN}

Proteína. En las quincenas 3, 6 y 7 posparto se presentó efecto $(p<0.05)$ del a mamantamiento sobre las concentraciones de proteína en suero sanguíneo (Tabla 2), se encontraron mayores concentraciones de proteína en los animales en amamantamiento experimental $(91.2,94.2$ y
$95.8 \mathrm{~g} / \mathrm{L}$ ) en comparación al tradicional (63.8, $71.1 \mathrm{y} 71.0 \mathrm{~g} / \mathrm{l})$. Estos resultados permiten afirmar que el amamantamiento restringido mejora el balance de proteína en sangre desde el parto hasta la quincena 7 posparto. En tanto que se podría presentar un balance negativo de proteína para los animales en amamantamiento tradicional, posiblemente 
determinado por una mayor producción de leche total en aquellas vacas que amamantan su cría, ocasionado por un mayor estímulo de succión (20). Las concentraciones de proteína en suero encontradas en el presente trabajo fueron superiores a las reportadas por la literatura (4, 21) particularmente en las vacas del amamantamiento experimental y suplementadas.
Las vacas suplementadas tuvieron mayores niveles de proteína $(p<0.05)$ solamente en la quincena 7 postparto en comparación con las vacas no suplementadas (Tabla 2 ), lo que podría ser explicado por el momento en el cual la vaca, bien sea de alta o baja producción, alcanza su equilibrio metabólico después del parto $(21,22)$.

Tabla 2. Efecto del amamantamiento y la suplementación sobre los niveles de proteína ( $g / l$ ) hasta la semana 14 a postparto.

\begin{tabular}{cccccccc}
\hline & Q 1 & Q 2 & Q 3 & Q 4 & Q 5 & Q 6 & Q 7 \\
S1 & 90,0 & 91,7 & 85,7 & 72,2 & 88,6 & 87,9 & $97,1 \mathrm{a}$ \\
S2 & 70,0 & 75,4 & 69,3 & 67,7 & 74,3 & 77,4 & $69,7 \mathrm{~b}$ \\
A1 & 83,7 & 93,9 & $91,2 \mathrm{a}$ & 74,1 & 91,7 & $94,2 \mathrm{a}$ & 95,8 \\
A2 & 76,3 & 73,2 & $63,8 \mathrm{~b}$ & 65,9 & 71,2 & $71,1 \mathrm{~b}$ & 95,8 \\
\hline
\end{tabular}

$\mathrm{Q}=\mathrm{Q}$ uincena, $\mathrm{S} 1$ = Suplemento. $\mathrm{S} 2$ = No suplementados, $\mathrm{A} 1=$ Amamantamiento experimental. $\mathrm{A} 2$ = Amamantamiento tradicional Para el tipo de amamantamiento, letras diferentes en la misma columna difieren estadísticamente $(p<0.05)$.

Para el tipo de suplementación, letras diferentes en la misma fila difieren estadísticamente $(p<0.05)$.

Urea. El amamantamiento y la suplementación no tuvieron efecto $(p>0.05)$ sobre las concentraciones de urea en suero sanguíneo desde el momento del parto hasta la quincena 7 postparto (Tabla 3) y no hubo interacción entre las categorías de los tratamientos en estudio. Resultados similares han sido comunicados por Doepel et al. (9) y Reist et al. (23) quienes encontraron que las concentraciones de urea en suero fueron similares para vacas utilizando diferentes tipos de suplementación proteica. Altas concentraciones de urea en sangre son un indicativo de incremento en la ureogénesis, como resultado de la oxidación de aminoácidos en exceso de aquellos requeridos para la síntesis proteica o a la detoxificación del amonio absorbido (24). Este fenómeno no se encontró en el presente estudio lo cual podría indicar que los niveles de suplementación no estuvieron por encima de los requeridos por los animales experimentales.

Tabla 3. Efecto del amamantamiento y la suplementación sobre los niveles de urea $(\mathrm{mml} / \mathrm{l})$ hasta la semana 14 a postparto.

\begin{tabular}{llllllll}
\hline & Q 1 & Q 2 & Q 3 & Q 4 & Q 5 & Q 6 & Q 7 \\
S1 & 6,7 & 5,8 & 5,8 & 5,1 & 5,9 & 6,3 & 5,4 \\
S2 & 5,1 & 5,4 & 5,2 & 5,1 & 3,8 & 4,4 & 4,8 \\
A1 & 4,8 & 5,0 & 5,7 & 6,4 & 4,4 & 4,2 & 4,2 \\
A2 & 7,0 & 6,1 & 5,2 & 6,4 & 5,3 & 6,5 & 6,1 \\
\hline
\end{tabular}

$\mathrm{Q}=\mathrm{Q}$ uincena, $\mathrm{S} 1$ = Suplemento. $\mathrm{S} 2=\mathrm{N}$ o suplementados, $\mathrm{A} 1=$ Amamantamiento experimental. $\mathrm{A} 2=$ Amamantamiento tradicional. Para el tipo de amamantamiento, letras diferentes en la misma columna difieren estadísticamente $(p<0.05)$.

Para el tipo de suplementación, letras diferentes en la misma fila difieren estadísticamente $(p<0.05)$.

BUN. No se presentó efecto de los tratamientos $(p>0.05)$ sobre el BUN desde el parto hasta la quincena 7 postparto (Tabla 4) y no hubo interacción entre los tratamientos. Los valores promedio del BUN durante el experimento fueron inferiores a $20 \mathrm{mg} / \mathrm{dl}$, valor a partir del cual se considera un balance negativo de energía, indicando por lo tanto que durante el período postparto no hubo desequilibrio energía-proteína, independiente de la cantidad consumida en relación con sus requerimientos. 
Tabla 4. Efecto del amamantamiento y la suplementación sobre los niveles de BUN (mg/dl) hasta la semana 14 a postparto.

\begin{tabular}{lccccccc}
\hline & Q 1 & Q 2 & Q 3 & Q 4 & Q 5 & Q 6 & Q 7 \\
S1 & 18,8 & 16,3 & 16,2 & 14,4 & 16,7 & 20,1 & 15,3 \\
S2 & 14,4 & 15,0 & 14,6 & 21,5 & 11,6 & 16,7 & 15,9 \\
A1 & 13,5 & 14,1 & 16,0 & 17,9 & 13,3 & 16,2 & 14,2 \\
A2 & 19,8 & 17,2 & 14,7 & 18,0 & 15,0 & 20,6 & 17,0 \\
\hline
\end{tabular}

$\mathrm{Q}=\mathrm{Q}$ uincena, $\mathrm{S} 1=$ Suplemento. $\mathrm{S} 2=\mathrm{N}$ o suplementados, $\mathrm{A} 1=$ Amamantamiento experimental. $\mathrm{A} 2=$ Amamantamiento tradicional

G lucosa. Como se observa en la tabla 5 , no se presentó efecto de los tratamientos $(p>0.05)$ sobre las concentraciones de glucosa en suero desde la quincenas 1 hasta la 7 postparto. Este resultado puede ser explicado dado que la glicemia no presenta grandes variaciones independientemente del nivel de alimentación, por estar bajo estricta regulación hormonal, (3, $9,21,25,26)$. Además, la glucosa no es tan sensible a los cambios en el balance energético como pueden ser otros indicadores (27).

En la quincena 5 , las vacas que recibieron semilla de algodón bajo el sistema de amamantamiento tradicional presentaron niveles de glucosa por debajo $(p<0.05)$ en comparación con los demás tratamientos. Este resultado posiblemente se deba al hecho de que la suplementación proteica favorezca la secreción de insulina, la cual, a su vez, ejerce una función hipoglicemiante que favorece la entrada de glucosa a la célula. En este sentido, Doepel et al. (9) encontraron altos niveles de insulina en vacas de leche cuando fueron alimentadas con dietas altas en proteína y energía comparadas con dietas de baja proteína y alta energía. Adicionalmente, en el amamantamiento tradicional se presenta una mayor extracción de leche por parte de la cría ocasionando de esta manera un mayor traslado de glucosa para la síntesis de la leche.

Tabla 5. Efecto del amamantamiento y la suplementación sobre los niveles de glucosa (mmi/l) hasta la semana 14 a postparto.

\begin{tabular}{llllllll}
\hline & Q 1 & Q 2 & Q 3 & Q 4 & $* *$ Q 5 & Q 6 & Q 7 \\
S1 & 3,2 & 2,5 & 2,6 & 2,6 & $\mathrm{~T} 1: 3,2 \mathrm{a}$ & 3,4 & 2,8 \\
S2 & 2,6 & 3,0 & 2,9 & 2,9 & $\mathrm{~T} 2: 1,9 \mathrm{~b}$ & 3,5 & 3,0 \\
A1 & 2,7 & 2,4 & 2,8 & 2,5 & $\mathrm{~T} 3: 2,5 \mathrm{a}$ & 3,7 & 3,1 \\
A2 & 3,1 & 3,1 & 2,7 & 3,1 & $\mathrm{~T} 4: 4,1 \mathrm{a}$ & 3,2 & 2,8 \\
\hline
\end{tabular}

$\mathrm{Q}=\mathrm{Q}$ uincena, $\mathrm{S} 1=$ Suplemento. $\mathrm{S} 2=\mathrm{N}$ o suplementados, $\mathrm{A} 1=$ Amamantamiento experimental. $\mathrm{A} 2=$ Amamantamiento tradicional Para el tipo de amamantamiento, letras diferentes en la misma columna difieren estadísticamente $(p<0.05)$. Para el tipo de suplementación, letras diferentes en la misma fila difieren estadísticamente $(p<0.05)$.

** Interacción Q 5

Colesterol. Es sabido que, los niveles de colesterol, por efecto de la lactancia, se incrementan en las primeras etapas de ésta, para posteriormente decaer, $(4,21,27$ - 29). Independientemente de los tratamientos, en el presente estudio (Tabla 6), los niveles de colesterol en sangre presentaron un incremento a lo largo del período analizado. No se encontró efecto $(p>0.05)$ del amamantamiento durante la observación. Igual comportamiento tuvo la suplementación hasta la quincena 6 , pues en la siguiente quincena, se presentó efecto $(p<0.05)$, manifestado en una mayor concentración del colesterol en la sangre en las vacas que consumieron semilla de algodón.

Los incrementos de los niveles de colesterol encontrados en este estudio, por efecto de la lactancia se explican en razón de una hipercolesterolemia como resultado de movilización de lípidos causado por el glucagón o por un incremento en la síntesis de lipoproteínas plasmáticas o también puede ser debido a una gran demanda energética mayor que la ofrecida en la dieta (30). 
Tabla 6. Efecto del amamantamiento y la suplementación sobre los niveles de colesterol $(\mathrm{mg} / \mathrm{dl})$ hasta la semana 14 a postparto.

\begin{tabular}{cccccccc}
\hline & Q 1 & Q 2 & Q 3 & Q 4 & Q 5 & Q 6 & Q 7 \\
S1 & 184,7 & 195,2 & 244,2 & 219,8 & 241,6 & 269,2 & $247,6 \mathrm{a}$ \\
S2 & 157,5 & 156,2 & 169,3 & 184,5 & 201,9 & 197,3 & $178,6 \mathrm{~b}$ \\
A1 & 134,1 & 163,5 & 191,5 & 180,7 & 206,5 & 207,1 & 207,6 \\
A2 & 208,1 & 187,9 & 222,1 & 223,6 & 237,0 & 259,3 & 218,5 \\
\hline
\end{tabular}

$\mathrm{Q}=\mathrm{Q}$ uincena, $\mathrm{S} 1$ = Suplemento. $\mathrm{S} 2$ = No suplementadoS, $\mathrm{A} 1$ = Amamantamiento experimental. A2 = Amamantamiento tradicional. Para el tipo de amamantamiento, letras diferentes en la misma columna difieren estadísticamente $(p<0.05)$.

Para el tipo de suplementación, letras diferentes en la misma fila difieren estadísticamente $(p<0.05)$.

Triglicéridos. Se presentó interacción $(p<0.05)$ entre el amamantamiento experimental y la suplementación en las quincenas 2 y 7 postparto (Tabla 7), reflejado en una mayor concentración de triglicéridos en sangre. Estos resultados podrían ser explicado por la alta concentración de lípidos en la semilla de algodón y por la ausencia de la cría en el tratamiento experimental. En este sistema se obtiene una menor extracción de leche con menor utilización de triglicéridos sanguíneos para la síntesis de la grasa de la leche. Los resultados concuerdan con los de otros autores (28, 31-33). Con respecto a la quincena 6 , se encontraron las mayores concentraciones de triglicéridos en el T4 en comparación con T1, T2 y T3, lo que podría obedecer a una mayor lipólisis, como consecuencia de procesos catabólicos, generados en la lactancia tardía (34).

Hubo efecto $(p<0.05)$ de la suplementación a base de semilla de algodón sobre las concentraciones de triglicéridos en la quincena 2 , reflejando de esta manera el efecto de gran contenido de lípidos presente en las semillas de algodón.

Tabla 7. Efecto del amamantamiento y la suplementación sobre los niveles de triglicéridos ( $\mathrm{mg} / \mathrm{dl}$ ) hasta la semana 14 a postparto.

\begin{tabular}{cccccccc}
\hline & Q 1 & Q 2 & Q 3 & Q 4 & Q 5 & Q 6 & Q 7 \\
S1 & 184,7 & 195,2 & 244,2 & 219,8 & 241,6 & 269,2 & 247,6 a \\
S2 & 157,5 & 156,2 & 169,3 & 184,5 & 201,9 & 197,3 & 178,6 b \\
A1 & 134,1 & 163,5 & 191,5 & 180,7 & 206,5 & 207,1 & 207,6 \\
A2 & 208,1 & 187,9 & 222,1 & 223,6 & 237,0 & 259,3 & 218,5 \\
\hline
\end{tabular}

$\mathrm{Q}=\mathrm{Q}$ uincena, $\mathrm{S} 1$ = Suplemento. $\mathrm{S} 2$ = No suplementadoS, $\mathrm{A} 1$ = Amamantamiento experimental. A2 = Amamantamiento tradicional. Para el tipo de amamantamiento, letras diferentes en la misma columna difieren estadísticamente $(p<0.05)$.

Para el tipo de suplementación, letras diferentes en la misma fila difieren estadísticamente $(p<0.05)$.

** Interacción Q 2 y diferencia en suplemento, interacción Q 6 y Q 7

Lipoproteínas de alta densidad (HDL). Con excepción de la quincena 4 , no se presentó efecto de la suplementación $(p>0.05)$ sobre los niveles de HDL en suero durante el período postparto (Tabla 8). En la quincena 4 los niveles de HDL en los animales suplementados alcanzaronniveles de $52.8 \mathrm{mg} / \mathrm{dl}$, marcadamente superiores $(p<0.05)$ a los niveles obtenidos en los animales no suplementados que alcanzaron $27.0 \mathrm{mg} / \mathrm{dl}$. Estos resultados coinciden con lo comunicado por Rayssiguier et al. (35) quienes sostienen que las concentraciones de HDL son bajas cerca del parto y aumentan hasta alcanzar valores más elevados 4 semanas postparto. Esta elevación del HDL avanzado el postparto puede ser explicada por ser esta lipoproteína la responsable del transporte de lípidos plasmáticos que se elevan por efecto de catabolismo lipídico en respuesta al aumento de la demanda energética y disminución del consumo de alimento en el primer tercio del postparto. 
Igualmente, a excepción de la quincena 5 , no se presentó efecto $(p>0.05)$ del amamantamiento sobre las concentraciones de HDL en suero. En la quincena 5 los niveles de $\mathrm{HDL}$ en el tratamiento tradicional fueron de $60.1 \mathrm{mg} / \mathrm{dl}$ superior $(p<0.05)$ a las concentraciones de HDL $(23.8 \mathrm{mg} / \mathrm{dl})$ en los animales sometidos al amamantamiento experimental. Lo que podría explicarse por un mayor catabolismo de lípidos por una mayor demanda de energía para la síntesis de la leche, dada la presencia de la cría, que ocasiona un mayor estímulo de succión.

Tabla 8. Efecto del amamantamiento y la suplementación sobre los niveles de HDL (mg/dl) hasta la semana 14 a postparto.

\begin{tabular}{cccccccc}
\hline & Q 1 & Q 2 & Q 3 & Q 4 & Q 5 & Q 6 & Q 7 \\
S1 & 35,2 & 40,8 & 54,4 & $52,8 \mathrm{a}$ & 48,8 & 45,2 & 51,2 \\
S2 & 25,6 & 27,9 & 28,7 & $27,0 \mathrm{~b}$ & 35,1 & 39,8 & 38,1 \\
A1 & 20,9 & 29,8 & 29,0 & 29,5 & $23,8 \mathrm{~b}$ & 32,7 & 36,4 \\
A2 & 39,9 & 39,0 & 54,0 & 50,3 & $60,1 \mathrm{a}$ & 52,3 & 52,9 \\
\hline
\end{tabular}

$\mathrm{Q}=\mathrm{Q}$ uincena, $\mathrm{S} 1$ = Suplemento. $\mathrm{S} 2$ = No suplementados, $\mathrm{A} 1=$ Amamantamiento experimental. $\mathrm{A} 2=$ Amamantamiento tradicional. Para el tipo de amamantamiento, letras diferentes en la misma columna difieren estadísticamente $(p<0.05)$.

Para el tipo de suplementación, letras diferentes en la misma fila difieren estadísticamente $(p<0.05)$.

Lipoproteínas de baja densidad (LDL). No se presentó efecto $(p>0.05)$ de la suplementación y del tipo de amamantamiento sobre las concentraciones de LDL (Tabla 9), indicando que la mayor parte de los lípidos plasmáticos en el bovino son transportados en forma de HDL, coincidiendo con lo reportado por Rayssiguier et al. (35) y Ceballos et al. (21).

Tabla 9. Efecto del amamantamiento y la suplementación sobre los niveles de LDL (mg/dl) hasta la semana 14 a postparto.

\begin{tabular}{llllllll}
\hline & Q 1 & Q 2 & Q 3 & Q 4 & Q 5 & Q 6 & Q 7 \\
S1 & 1,2 & 1,5 & 1,9 & 1,8 & 2,3 & 2,2 & 1,7 \\
S2 & 1,7 & 1,9 & 2,0 & 2,2 & 2,0 & 2,1 & 2,1 \\
A1 & 1,3 & 1,6 & 1,9 & 2,1 & 2,2 & 2,3 & 1,9 \\
A2 & 1,7 & 1,7 & 1,9 & 2,0 & 2,0 & 1,9 & 1,9 \\
\hline
\end{tabular}

$\mathrm{Q}=\mathrm{Q}$ uincena, $\mathrm{S} 1$ = Suplemento, $\mathrm{S} 2=\mathrm{N}$ o suplementados, $\mathrm{A} 1=$ Amamantamiento experimental, $\mathrm{A} 2=$ Amamantamiento tradicional. Para el tipo de amamantamiento, letras diferentes en la misma columna difieren estadísticamente $(p<0.05)$.

Para el tipo de suplementación, letras diferentes en la misma fila difieren estadísticamente $(p<0.05)$.

En conclusión, la suplementación con semilla de algodón no tuvo efecto sobre los perfiles metabólicos durante el preparto. En el postparto la suplementación y el amamantamiento tuvieron efecto en algunos metabolitos, principalmente triglicéridos y proteínas.

\section{Agradecimientos}

A la oficina administradora de investigación y extensión de la Universidad de Córdoba (CIUC) por la financiación del trabajo; al Doctor Álvaro Cabrales, propietario de la Hacienda Canta Rana, por permitir la realización del estudio. 


\section{REFERENCIAS}

1. G rummer RR. Impact of changes in organic nutrient metabolism on feeding the transition dairy cow. J Anim Sci 1995; 73: 2820-2833.

2. McNamara S, O 'Mara FP, Rath M, Murphy J). Effects of Different Transition Diets on Dry Matter Intake, Milk Production, and Milk Composition in Dairy Cows. J Dairy Sci 2003; 86: 2397-2408.

3. Greenfield RB, Cecava MJ, Johnson TR, Donkin SS. Impact of Dietary Protein Amount and Rumen Undegradability on Intake, Peripartum Liver Triglyceride, Plasma Metabolites and Milk Production in Transition Dairy Cattle. J Dairy Sci 2000; 83: 703-710.

4. Cavestany D, Blanc JE, Kulcsar M, U riarte G, C hilibroste P, M eikle A, et al. Studies of the Transition Cow Under a Pasture-based Milk Production System: Metabolic Profiles. J Vet Med 2005; 52: 1-7.

5. Grummer RR. Etiology of lipid-related metabolic disorders in periparturient dairy cows. J Dairy Sci 1993; 76: 3882-3896.

6. Goff JP, Horst RL. Physiological changes at parturition and their relationship to metabolic disorders. J Dairy Sci 1997; 80: 1260-1268.

7. Putnam DE, Varga GA. Protein density and its influence on metabolite concentration and nitrogen retention by Holstein cows in late gestation. J Dairy Sci 1998; 81: 16081618.

8. Van Saun RJ, Idleman SC, Sniffen CJ. Effect of undegradable protein amount fed prepartum on postpartum production in first lactation Holstein cows. J Dairy Sci 1993; 76: 236-244.

9. Doepel L, Lapierre H, Kennelly JJ. Peripartum Performance and M etabolism of Dairy Cows in Response to Prepartum Energy and Protein Intake J Dairy Sci 2002; 85: 2315-2334.
10. Schlink AC, G ibson DS, Liang ZJ, Dixon R. Calf management strategies and reproductive performance in a northern Australian cattle herd. Proc Aust Soc Anim Prod 1988; 17: 326-329.

11. Sullivan RM, O Rourke PK, Robertson DL, Cooke D. Effects of once-yearly Weanyng on some aspects of herd productivity in an extensive herd in the semi-arid tropics of northern Australia. Aust J Exp Agr 1992; 32: 149-156.

12. Das SM, Forsberg M, Wiktorsson $H$. Influence of restricted suckling and level of feed supplementation on postpartum reproductive performance of zebu and crossbred cattle in the semi-arid tropics. Acta Vet Scand 1999; 40: 57-67.

13. Short RE. Effects of suckling and mastectomy on bovine postpartum reproduction. J Anim Sci 1972; 34: 70-74.

14. Rayner IH, Edmunds J, Stokde J. Comparison of dairy and multiple sucled calf production with calf acess restricted. Aust J Exp Agr Anim Husb 1977; 17: 728-734.

15. Ferreira A. Efeito da amamantamentacao e do nivel nutricional na atividae ovariana de vacas mestizas leiteiras [tesis docto ral]. Universidad Federal de Vicosa, Minas Gerais, Brasil 1990.

16. Mancio A, HernandeZ F, Fonseca F. Efeito da amamentacao controlada ou interompida sobre 0 desempenho reproductivo de vacas de corte. Revista Medica Zootecnia 1998; 50: 765-770.

17. Mancio A, HernandeZ F, Fonseca F. Amamentacao controlada no desempenho reproductivo de vacas de corte. Arq Bras Vet Zootec 1999; 51: 79-84.

18. Perez-Hernández Garcia-Winder M, G allegos-Sanchez J. Postpartum anoestrus is reduced by increasing the within-day milking to suckling interval in dual purpose cows. Anim Reprod Sci 2002; 73: 159-68. 
19. Sanz A, Casasús I, Villalba D, Revilla R. Effects of suckling frequency and reed on productive performance, follicular dynamics and postpartum interval in beef cows. Anim Reprod Sci 2003; 79: 57-69.

20. Hoffman D, Stevenson J, Milton J. Restricting calf presence without suckling compared with weaning prolongs postpartum anovulation in beef cows. J Anim Sci 1996; 74: 190-198.

21. Ceballos A, Gómez PM, Velez MI, Villa NA, Lopez LF. Variación de los indicadores bioquímicos del balance de energía según el estado productivo en bovinos lecheros de Manizales, Colombia. Rev Col Cienc Pec 2002; 15: 1.

22. Valoyes HB. Efecto de la suplementación con salvado de arroz en la interacción nutrición-reproducción en vacas bajo el sistema de doble propósito. Tesis de $G$ rado. Facultad de Medicina Veterinaria y Zootecnia. Universidad de Córdoba, Montería, Colombia. 1994.

23. Reist M, Blum JW, Küpfer U. Time empty and ketone body status in the early postpartum period of dairy cows. Reprod Domest Anim 2003; 38: 41-49.

24. Lobley GE, Milano GD, Van Der Walt JG. The liver: Integrator of nitrogen metabolism. in Ruminant Physiology: Digestion, Metabolism, Growth, and Reproduction. Editor P. B. Cronje. 2000; p149-168.

25. Harrison RO, Ford SP, Young JW, Conley AJ, Freeman AE. Increased milk production versus reproductive and energy status of high producing dairy cows. J Dairy Sci 1990; 73: 2749-2758.

26. Bertics SJ, G rummer RR, Cadorniga-Valino $C$, Stoddard EE. Effect of prepartum dry matter intake on liver triglyceride concentration and early lactation. J Dairy Sci 1992; 75: 1914-1922.

27. Whitaker DA, Kelly JM. The use and interpretation of metabolic profiles in dairy cows. I Curso de Divulgación en Técnicas de RIA y Evaluación de Metabolitos Sanguíneos y Cinéticas Digestivas Relacionadas en la Nutrición y Reproducción en Bovinos. Maracay, Venezuela, 1994.

28. Bell, A. W. Regulation of organic nutrient metabolism during transition from late pregnancy to early lactation. J Anim Sci 1995; 73: 2804-2819.

29. Rukkwamsuk T, Kruip TAM, Meijer GAL, Wensing T. Hepatic fatty acid composition in periparturient dairy cows with fatty liver induced by intake of a high energy diet in the dry period. J Dairy Sci 1999; 82: 280 287.

30. Margolles E. Metabolitos sanguíneos en vacas altas productoras durante la gestación-lactancia en las condiciones de cuba y su relación con trastornos del metabolismo. Rev Cub Cienc Vet 1983; 10: 228-235.

31. Sommer $\mathrm{H}$. The role of the metabolic profile test in the control of cattle feeding. Magyar Állatorvosok Lapja. En: Memorias Segundo Seminario Internacional y metabolismo de la vaca lechera. Universidad de Caldas. Manizales, Colombia. 1999; p.714-717.

32. Calamari L, Bertoni G, Maiati MG, Cappa V. Sull' utilità di nuovi parametric ematochimici nella valutazione del profilo metabolico delle lattifere. Zoot N utri Anim 1989; Anno XV n. 3.

33. Wittwer FG, Tadich N, Blowey R. Variaciones en las concentraciones de algunos parámetros sanguíneos en vacas productoras de leche durante las primeras semanas de lactancia. Vet M éx 1983; 14: 1983.

34. Basoglu A, Sevine M, OK M, Gokeen M. Peri- and postparturient concentrations of lipid protein, insulin and glucose in normal dairy cow. Turk-Veterinerlik-ve-HayvancilikDergisi. 1998; 22: 141-144.

35. Rayssiguier Y, Mazur A, Gueux E. Plasma lipoprotein and fatty liver in dairy cows. Res Vet Sci 1988; 45: 389-393. 